\title{
Helicobacter Pylori Seropositivity in Hyperemesis Gravidarum During Pregnancy
}

Esmail El-Garhy, Yehia A. Wafa, Ahmed Okasha

Department of Obstetrics \& Gynecology, Faculty of Medicine, Al-Azhar University

Corresponding author: Ahmed Okasha; Mobile: +201005087129; Email: docahmedokasha@gmail.com

\begin{abstract}
Background: Prevalence of hyperemesis gravidarum varies from 0.3 to $1.5 \%$ of all live births. The exact cause is not well known and is probably multifactorial. It is the most common cause of hospitalization in the first half of pregnancy and second only to preterm labor for pregnancy overall. The etiology of emesis gravidarum remains unknown. But a number of possible causes have been studied as endocrinal, immunological, psychological, metabolic, genetic and even infectious such as helicobacter pylori infection.
\end{abstract}

Aim of the Work: To assess the value of screening for helicobacter pylori seropositivity in hyperemesis gravidarum for better evaluating and improving the cure rate especially in resistant cases.

Patients and methods: A prospective controlled comparative study was conducted on 100 pregnant women in the first trimester, where 50 of them were suffering from hyperemesis gravidarum (group A) and another 50 healthy women were chosen as a control group (group B). They were recruited from the outpatient clinic of Al-Galaa Maternity Teaching Hospital, Cairo, Egypt from January 2019 till June 2019. After approval of the local ethics committee, a written consent was obtained from each woman before inclusion in the study. Fasting and post prandial sugar, Liver and kidney function tests, thyroid function tests, CBC, urine and electrolyte examination as well as serum examination for IgG of helicobacter pylori were done for each one.

Results: Serum helicobacter pylori IgG antibodies seropositivity and acetonuria was significantly higher in group A than in group B while serum sodium and potassium levels were significantly lower in patients with hyperemesis gravidarum than control group.

Conclusion: The treatment of $H$. Pylori infection may reduce the risk of hyperemesis gravidarum and its complications. Keywords: Hyperemesis gravidarum, Helicobacter pylori, Morning sickness.

\section{INTRODUCTION}

Nausea and vomiting during pregnancy, which is also known as 'morning sickness', affects between $70-80 \%$ of all pregnancies in the first trimester. Usually, it starts between 4 and 8 weeks of gestation, however the symptoms might continue till the $16^{\text {th }}-18^{\text {th }}$ week. It is generally a mild condition and self-limited. A small number of pregnant women have a more acute sequalae, with the most severe form known as hyperemesis gravidarum (1). In almost $1-10 \%$ of pregnancies, symptoms may continue till 20-22 weeks ${ }^{(2)}$. Hyperemesis gravidarum (HG) is characterized by persistent nausea and vomiting accompanied by ketosis and loss of weight ( $>5 \%$ of the weight before pregnancy). It may cause hypovolemia, electrolytes disturbance and acid-base imbalance, nutritional deficiencies, and even - in severe cases death. Severe cases with hyperemesis require hospitalization in $0.3-2 \%$ of pregnancies ${ }^{(3)}$.

Prevalence of hyperemesis gravidarum varies from 0.3 to $1.5 \%$ of all live births. ${ }^{(4)}$ The exact etiology of hyperemesis gravidarum is not well known and is probably multifactorial in which psychological factors, disturbance of gastrointestinal motility, hormonal changes, infections, immunological, metabolic and anatomical factors appear to intervene ${ }^{(5)}$. It is the most common cause of hospitalization in the first half of pregnancy and second only after preterm labor for pregnancy overall. It can be associated with serious maternal morbidity such as Wernicke's encephalopathy and fetal morbidity such as intrauterine growth retardation, and in severe cases maternal and fetal death may happen ${ }^{(4)}$.

The Helicobacter pylori (H. pylori) is considered as an important cause of gastritis in human beings and as an essential factor in the pathogenesis of peptic ulcer. Many findings suggest that Helicobacter pylori is also involved in the pathogenesis of cancer and lymphoma of the stomach ${ }^{(6)}$.

In developing countries, $70 \%$ to $90 \%$ of the population is infected by the bacteria, while in industrialized countries the prevalence is smaller, ranging between $25 \%$ and $50 \%{ }^{(4)}$.

Many methods of Helicobacter pylori testing exist. Noninvasive tests for Helicobacter pylori infection include the blood antibody test, the stool antigen test, or with the carbon urea breath test (in which the patient drinks ${ }^{14} \mathrm{C}$ or ${ }^{13} \mathrm{C}$ labelled urea, then the bacterium metabolizes the labelled urea producing labelled $\mathrm{CO}_{2}$ that can be detected in the breath of the patient). The other method for Helicobacter pylori infection detection is endoscopic biopsy check with a rapid urease test, histological examination, and microbial culture ${ }^{(7)}$.

In essence, serology samples the whole stomach whereas biopsy only samples a small portion, and the inflammatory process may be patchy, so serologic analysis is considered more sensitive than diagnostic methods involving biopsy. The development of Helicobacter pylori-specific fluorescent serum antibody test helps for easy and suitable screening for $\mathrm{H}$. pylori infection and because of its simple, cheap and non- 
invasive testing, it becomes possible to detect • Helicobacter pylori infection in pregnant women ${ }^{(8)}$.

\section{AIM OF THE WORK}

To assess the value of screening for Helicobacter pylori seropositivity in hyperemesis gravidarum for better evaluating the condition and improving the cure rate especially in resistant cases of hyperemesis gravidarum.

\section{PATIENTS AND METHODS}

\section{Type of the study:}

A prospective controlled comparative study to assess the value of screening Helicobacter pylori seropositivity in hyperemesis gravidarum was conducted on 100 pregnant women in the first trimester, where 50 of them were suffering from hyperemesis gravidarum (group A) and another 50 healthy women were chosen as a control group (group B). They were recruited from the outpatient clinic of Al-Galaa Maternity Teaching Hospital, Cairo, Egypt from January 2019 till June 2019.

\section{Ethical approval:}

After approval of ethical and research committee of council of Obstetrics and Gynecology Department, Al-Azhar University,

a written consent was obtained from each woman after explanation of the study purpose.

Women suffering from other causes of vomiting in early pregnancy were excluded.

\section{Inclusion Criteria:}

- Age: 18 - 40 years old.

- Gestational age: less than 16 weeks confirmed by U/S.

- Diagnosis of hyperemesis gravidarum according to H.E.R. Foundation (Hyperemesis Education \& Research Foundation) is based on:

- excessive pregnancy-related nausea and/or vomiting that prevents adequate intake of food and fluids.

- Measuring weight loss (>5\% of prepregnancy weight).

- Signs of dehydration:

- Ketonuria (+1 or more).

- Hemoconcentration.

\section{Exclusion Criteria:}

- Multiple gestation pregnancy.

- Hydatidiform molar pregnancy.

- Other causes of vomiting such as:

- Gastroenteritis.

- Cholecystitis.

- Pyelonephritis.
Liver dysfunction .

Urinary tract infection .

\section{All cases were subjected to the following:}

- Full history taking.

- Clinical examination that includes general and abdominal examinations.

- Transabdominal or Transvaginal pelvic ultrasound: To assess fetal viability and confirm the gestational age and to exclude gestational trophoblastic diseases and multiple gestations.

- Routine laboratory investigations.

1. Complete blood count: (RBCs, WBCs, Platelet count, $\mathrm{Hb} \%$, Hemoglobin concentration): dehydration causes a raised hemoglobin concentration and hematocrit value.

2. Complete urine analysis: for protein, ketone bodies, bile salts, and sample sent for culture to exclude urinary tract infection which may be the cause of the vomiting.

3. Serum electrolytes: (sodium, potassium) for detection of electrolyte disturbance if present.

4. Renal function tests: (serum creatinine, creatinine clearance) to exclude renal dysfunction.

5. Liver function tests: (SGOT and SGPT) to exclude liver dysfunction.

6. Thyroid function tests: (TSH, Free T3, Free T4) to exclude hyperthyroidism.

7. Fasting and 2 hours post prandial sugar to exclude diabetic ketoacidosis.

- Five ml venous blood sample was collected to test the serum for Helicobacter pylori IgG seropositivity by Enzyme Linked Immunoassay (ELISA).

\section{Statistical analysis:}

Recorded data were analyzed using the statistical package for social sciences, version 20.0 (SPSS Inc., Chicago, Illinois, USA). Quantitative data were expressed as mean \pm standard deviation (SD). Qualitative data were expressed as frequency and percentage.

\section{The following tests were done:}

- Independent-samples t-test of significance was used when comparing between two means.

- Chi-square $\left(\mathrm{x}^{2}\right)$ test of significance was used in order to compare proportions between qualitative parameters.

- The confidence interval was set to $95 \%$ and the margin of error accepted was set to 5\%. So, the pvalue was considered significant as the following: $*$ P-value $<0.05$ was considered significant. $* *$ P-value $<0.001$ was considered as highly significant.

P-value $>0.05$ was considered insignificant. 


\section{RESULTS}

The results of the present study are demonstrated in the following tables

Table (1): Basic characteristics of the studied groups

\begin{tabular}{|c|c|c|c|c|c|}
\hline & & $\begin{array}{l}\text { Hyperemesis gravidarum } \\
\text { group }(\mathbf{A})(n=50)\end{array}$ & $\begin{array}{l}\text { Control group }(B) \\
\quad(n=50)\end{array}$ & \multicolumn{2}{|c|}{ Independent t-test } \\
\hline & & Mean \pm SD (Range) & Mean \pm SD (Range) & $\mathbf{T}$ & p-value \\
\hline \multicolumn{2}{|c|}{ Maternal age (years) } & $27.9 \pm 5.89$ & $26.26 \pm 5.74$ & 1.41 & 0.162 \\
\hline \multicolumn{2}{|c|}{ Gestational age (weeks) } & $8.64 \pm 2.27$ & $8.52 \pm 2.1$ & 0.274 & 0.784 \\
\hline \multicolumn{2}{|c|}{ BMI (kg/m2) } & $22.66 \pm 1.91$ & $22.97 \pm 1.75$ & 0.854 & 0.395 \\
\hline \multirow{2}{*}{ Parity } & $\mathrm{PG}$ & $27(54 \%)$ & $19(38 \%)$ & \multirow{2}{*}{2.576} & \multirow{2}{*}{0.108} \\
\hline & MG & $23(46 \%)$ & $31(62 \%)$ & & \\
\hline
\end{tabular}

t-Independent sample t-test; $p$-value >0.05 NS; *p-value <0.05 S; **p-value <0.001 HS

This table shows no significant differences were reported between the both groups.

Table (2): Basic laboratory records of studied groups

\begin{tabular}{|c|c|c|c|c|}
\hline \multirow[t]{2}{*}{ NM } & $\begin{array}{c}\text { Hyperemesis Gravidarum } \\
\text { group (A) (no=50) }\end{array}$ & $\begin{array}{c}\text { Control group }(B) \\
(n o=50)\end{array}$ & \multirow[t]{2}{*}{$\mathbf{T}$} & \multirow[t]{2}{*}{$\mathbf{P}$} \\
\hline & Mean \pm SD (Range) & Mean \pm SD (Range) & & \\
\hline $\mathrm{HB}(\mathrm{gm} \%)$ & $\begin{array}{c}10.84 \pm 0.74 \\
9.8-12.1\end{array}$ & $\begin{array}{c}11.05 \pm 0.78 \\
9.9-12.0\end{array}$ & -1.415 & 0.160 \\
\hline Albumin (gm/dl) & $\begin{array}{c}4.12 \pm 0.35 \\
3.6-4.6\end{array}$ & $\begin{array}{c}4.18 \pm 0.25 \\
3.6-4.7\end{array}$ & -0.450 & 0.654 \\
\hline Total bilirubin (mg/dl) & $\begin{array}{c}1.99 \pm 0.17 \\
1.7-2.3\end{array}$ & $\begin{array}{c}1.97 \pm 0.22 \\
1.3-2.3\end{array}$ & 0.500 & 0.618 \\
\hline Creatinine (mg/dl) & $\begin{array}{c}0.82 \pm 0.15 \\
0.6-1.1\end{array}$ & $\begin{array}{c}0.82 \pm 0.12 \\
0.6-1.1\end{array}$ & -0.213 & 0.832 \\
\hline NA (mmol/L) & $\begin{array}{c}131.12 \pm 2.3 \\
130-142\end{array}$ & $\begin{array}{c}141.48 \pm 3.12 \\
135-146\end{array}$ & 18.899 & $<0.001^{*}$ \\
\hline $\mathrm{K}(\mathrm{mmol} / \mathrm{L})$ & $\begin{array}{c}2.92 \pm 0.36 \\
2.5-3.8\end{array}$ & $\begin{array}{c}4.22 \pm 0.43 \\
3.7-5.1\end{array}$ & 16.391 & $<0.001 *$ \\
\hline
\end{tabular}

t-Independent sample t-test; *p-value <0.05 S. This table shows that serum sodium and potassium levels were significantly lower in patients with hyperemesis gravidarum than control group. However, other laboratory parameters were non- significantly different between both groups.

Table (3): Acetonuria and albuminuria in studied groups.

\begin{tabular}{|c|c|c|c|c|c|c|c|}
\hline & & & \begin{tabular}{|c|}
$\begin{array}{c}\text { Hyperemesis Gravidarum } \\
\text { group }(\mathrm{A})(\mathrm{no}=\mathbf{5 0})\end{array}$ \\
\end{tabular} & $\begin{array}{l}\text { Control group } \\
\text { (B) }(\text { no=50) }\end{array}$ & Total & $\mathrm{X} 2$ & $\mathbf{P}$ \\
\hline \multirow{8}{*}{ Acetone } & \multirow{2}{*}{ Nil } & $\mathrm{N}$ & 9 & 45 & 54 & \multirow{8}{*}{55.3} & \multirow{8}{*}{$<0.001 *$} \\
\hline & & $\%$ & $18.0 \%$ & $90.0 \%$ & $54.0 \%$ & & \\
\hline & \multirow{2}{*}{+} & $\mathrm{N}$ & 15 & 5 & 20 & & \\
\hline & & $\%$ & $30.0 \%$ & $10.0 \%$ & $20.0 \%$ & & \\
\hline & \multirow{2}{*}{++} & $\mathrm{N}$ & 16 & 0 & 16 & & \\
\hline & & $\%$ & $32.0 \%$ & $0.0 \%$ & $16.0 \%$ & & \\
\hline & \multirow{2}{*}{+++} & $\mathrm{N}$ & 10 & 0 & 10 & & \\
\hline & & $\%$ & $20.0 \%$ & $0.0 \%$ & $10.0 \%$ & & \\
\hline \multirow{8}{*}{ Albumin } & \multirow{2}{*}{ Nil } & $\mathrm{N}$ & 23 & 45 & 68 & \multirow{8}{*}{23.66} & \multirow{8}{*}{$k 0.001 *$} \\
\hline & & $\%$ & $46.0 \%$ & $90.0 \%$ & $68.0 \%$ & & \\
\hline & \multirow{2}{*}{+} & $\mathrm{N}$ & 17 & 5 & 22 & & \\
\hline & & $\%$ & $34.0 \%$ & $10.0 \%$ & $22.0 \%$ & & \\
\hline & \multirow{2}{*}{++} & $\mathrm{N}$ & 4 & 0 & 4 & & \\
\hline & & $\%$ & $8.0 \%$ & $0.0 \%$ & $4.0 \%$ & & \\
\hline & \multirow{2}{*}{+++} & $\mathrm{N}$ & 6 & 0 & 6 & & \\
\hline & & $\%$ & $12.0 \%$ & $0.0 \%$ & $6.0 \%$ & & \\
\hline \multirow{2}{*}{\multicolumn{2}{|c|}{ Total }} & $\mathrm{N}$ & 50 & 50 & 100 & & \\
\hline & & $\%$ & $100.0 \%$ & 100.0 & $100.0 \%$ & & \\
\hline
\end{tabular}

t-Independent sample t-test; $p$-value $>0.05 \mathrm{NS}$; *p-value <0.05 S. This table shows acetonuria was statistically significant in hyperemesis group $(41 / 50=82 \%)$ compared to control healthy one $(5 / 50=10 \%)$. Moreover, the reported acetonuria in the control group was only mild. On other hand, the reported acetonuria in hyperemesis group was mild $(15 / 50=30 \%)$, moderate $(16 / 50=32 \%)$ and severe $(10 / 50=20 \%)$. In addition, Albuminuria was reported significantly more in hyperemesis group $(27 / 50=54 \%)$, compared to $(5 / 50=10 \%)$ in control group. Only mild degree of albuminuria was reported in control group. On the other hand, mild $(17 / 50=34 \%)$, moderate $(4 / 50=8 \%)$, and severe $(6 / 50=12 \%)$ degrees of albuminuria were reported in hyperemesis. 
Table (4): H. pylori IgG antibody seropositivity among studied groups.

\begin{tabular}{|c|c|c|c|c|c|c|}
\hline & & & \begin{tabular}{|c} 
Hyperemesis \\
Gravidarum group \\
$(\mathrm{A})$ \\
$(\mathrm{no}=\mathbf{5 0})$
\end{tabular} & $\begin{array}{c}\text { Control group } \\
(\mathrm{B}) \\
(\mathrm{no}=50)\end{array}$ & $\mathrm{X} 2$ & $\mathbf{P}$ \\
\hline \multirow{4}{*}{$\begin{array}{l}\text { H. Pylori } \\
\text { N (\%) }\end{array}$} & \multirow{2}{*}{ Negative } & $\mathrm{N}$ & 6 & 35 & \multirow{4}{*}{34.766} & \multirow{4}{*}{$0.001 *$} \\
\hline & & $\%$ & $12 \%$ & $70 \%$ & & \\
\hline & \multirow{2}{*}{ Positive } & $\mathrm{N}$ & 44 & 15 & & \\
\hline & & $\%$ & $88 \%$ & $30 \%$ & & \\
\hline
\end{tabular}

t-Independent sample t-test;

$p$-value $>0.05 \mathrm{NS} ; *$ p-value $<0.05 \mathrm{~S}$

This table shows that serum helicobacter pylori IgG antibodies seropositivity was significantly different between hyperemesis group (88\%) and control group (30\%).

\section{DISCUSSION}

Nausea and vomiting of pregnancy (NVP), which is also known as "morning sickness" affects nearly $70 \%$ to $80 \%$ of all pregnancies in the first trimester. It starts typically between the $4^{\text {th }}$ and the $8^{\text {th }}$ week of gestation, with symptoms that can persist till 16-18 weeks ${ }^{(9)}$.

NVP is generally considered a mild condition and self-limited that may be controlled and treated conservatively. A small percentage of pregnant women have a more complicated course, with the most severe form of NVP known as hyperemesis gravidarum. Hyperemesis gravidarum may have negative effect on both maternal and fetal health. ${ }^{(10)}$

The exact etiology of hyperemesis gravidarum is unknown. However, there are many biological and physiological theories to explain the pathogenesis of hyperemesis gravidarum. Gestational hormone levels abnormalities, psychological problems before pregnancy and gastric motility disturbances in early pregnancy are the most common explaining theories ${ }^{(11)}$.

Studies as Wu et al. ${ }^{(12)}$, Hayakawa et al. ${ }^{(13)}$, and Karaca et al. (14), suggested that chronic Helicobacter pylori infection may play a role in hyperemesis gravidarum. Helicobacter pylori seropositivity was present in up to $60 \%$ of pregnant women compared with 50\% in the general population.

Helicobacter pylori infection was found in cases of persistent vomiting in pregnancy who were not responding to supportive treatment. However, endoscopic diagnosis should not be performed unless initial non-invasive tests are negative ${ }^{(15)}$.

Our study included 100 pregnant females in their first trimester of pregnancy.

Fifty of them were suffering from hyperemesis gravidarum according to diagnostic criteria mentioned in the inclusion criteria of the study (group A), other 50 pregnant females were healthy and presenting as the control group (group B).
In this study, we aimed to find the relation between Helicobacter pylori and hyperemesis gravidarum.

The basic characteristics of the studied groups (case and control) were comparable with no significant difference(table 1).

Serum sodium and potassium were lower in the hyperemesis group $(2.92 \pm 0.36$ and 131.12 \pm 2.3$)$ compared to the control group $(4.22 \pm 0.43$ and $141.48 \pm 3.12$ ), respectively, (table 2). These results agree with the results reported by Jarraya et al. ${ }^{(16)}$ and Li et al ${ }^{(17)}$.

Hypokalemia and hyponatremia in hyperemesis group, reported in this study, may be due to persistent and repeated vomiting with subsequent loss of sodium and potassium ions ${ }^{18}$.

Three Cases with severe hypokalemia $(\mathrm{K}<3.0 \mathrm{meq} / \mathrm{l})$ were admitted to ICU for intravenous potassium therapy. Other cases with less severe hypokalemia $(\mathrm{K}>3.0 \mathrm{meq} / \mathrm{l})$ were corrected by oral potassium syrup after controlling of their hyperemesis state by antiemetics.

Moreover, acetonuria was significantly more in the hyperemesis group compared to the control group, (table 3). These results agree with the results reported by Sheehan ${ }^{(19)}$, Goodwin ${ }^{(18)}$ and Fejzo ${ }^{(20)}$.

Acetonuria in the hyperemesis group may be because the body shifts to fat metabolism with increased production of ketone bodies (the metabolic end products of fat metabolism), and then these ketone bodies are excreted in urine. This shift towards fat metabolism is due to unavailability of sufficient or enough carbohydrates resulting from excessive and protracted vomiting ${ }^{(21)}$.

Positive serum IgG concentrations were found in 44 / 50 of hyperemesis patients (88\%) compared with 15 / 50 controls $(30 \%)$. This showed a significant statistical difference between hyperemesis group and control group, (table 4). 
Egyptian studies showed a significant association between Helicobacter pylori infection and hyperemesis gravidarum.

A case control study was done by Mai $\boldsymbol{e t}$ al. on Egyptian hyperemesis patients and healthy pregnant ladies. It showed a significant association between Helicobacter pylori infection and hyperemesis gravidarum, where $84.4 \%$ were $\mathrm{H}$. pylori positive and $15.6 \%$ were negative.

So, there is a significant correlation between Helicobacter pylori infection and occurrence of emesis and hyperemesis gravidarum. Several reports such as Frigo et al ${ }^{(21)}$, Jacoby and Porter. ${ }^{(22)}, \mathrm{Wu}$ et al..$^{(12)}$, Kazerooni et al. ${ }^{(8)}$, Haim Shirin et al. ${ }^{(23)}$ had found that there was association between hyperemesis gravidarum and Helicobacter pylori infection.

Results showed that there were a strong association between $\mathrm{H}$ pylori and hyperemesis gravidarum, allowing us to conclude that a test for Helicobacter pylori seropositivity should be done when a pregnant patient is presented with hyperemesis gravidarum.

There is a great controversy about the role of Helicobacter pylori infection and emesis gravidarum. About 32 articles between 1998 and 2014 met the inclusion criteria and their characteristics. There were 13 studies that found no association between Helicobacter pylori infection and the hyperemesis gravidarum.

On the other hand, 19 researches suggested the Helicobacter pylori infection during pregnancy might be a risk factor for pregnant women with hyperemesis gravidarum.

This result was similar to those studies reported the relationship between hyperemesis gravidarum and Helicobacter pylori infection, which is supported by reports of five cases of women who were diagnosed with hyperemesis gravidarum who did not respond to the standard hyperemesis gravidarum management, but they responded to Helicobacter pylori treatment, which resulted in a complete relief of symptoms and observed improvement ${ }^{(22)}$.

Helicobacter pylori can be transmitted mainly within families in the developed countries but can also be acquired from the community in the developing nations ${ }^{(23)}$

Helicobacter pylori can be transmitted through the contaminated food or water. Different epidemiologic studies had reported that the major risk factor of Helicobacter pylori infection is low socioeconomic level ${ }^{(23)}$.

Cases and controls in this study were recruited from the clinics of EL-Galaa Teaching Hospital where medical service is almost free. This explains the low socioeconomic level of all women participated in this study.
Patients' education for food safety was important. Careful food handling and hand washing were important to prevent transmission of foodborne pathogens to the diet of pregnant women. Patients' education for food safety should be considered to prevent the increasing numbers of infected cases especially in developing countries with low socioeconomic levels.

Further case reports described that there was significant improvement in patients with hyperemesis gravidarum and a positive Helicobacter pylori serum titer after treatment with clarithromycin and/or amoxicillin and a proton pump inhibitor (lansoprazole or omeprazole) or $\mathrm{H} 2$-antagonists (famotidine or ranitidine).

The advantages of the antibody tests were their low cost, widespread availability, and rapid results ${ }^{(24)}$.

Early detection for Helicobacter pylori and proof of eradication are the keys for preventing severe consequences of infection.

In the present study a serum anti- Helicobacter pylori $\mathrm{IgG}$ antibody by ELISA > 1.1 was associated with hyperemesis gravidarum at a sensitivity of $86.67 \%$, a specificity of $65.91 \%$, a positive predictive value of $72.22 \%$, a negative predictive value of 82.86 .

\section{CONCLUSION}

The results of this study showed that there is a strong association between Helicobacter pylori infection and hyperemesis gravidarum. These results agree with the results of many previous studies that take into consideration the complex nature of etiological factors of both hyperemesis gravidarum and Helicobacter pylori infection.

We recommend that the diagnostic test for Helicobacter pylori should be a part of hyperemesis gravidarum investigations, when patients are resistant to the standard therapy as well as in women who have the desire to become pregnant in the near future. especially it is easy, fast and accurate screening tool.

In longstanding conditions of hyperemesis gravidarum that are resistant to the standard management and the cases that extend to second trimester. Non teratogenic regimen for treatment of Helicobacter pylori may be considered in intractable cases.

Resistant cases of hyperemesis gravidarum should be treated with anti-Helicobacter pylori antibiotics. The best for treatment is combination therapy of Amoxicillin (category A) $750 \mathrm{mg}$ TID and Metronidazole (category B/C) $500 \mathrm{mg}$ QID, combined with or given after anti-secretory drug therapy (PPIs) with meals for two weeks. This gives success rate $85 \%$. It is considered the safest method for treatment of Helicobacter pylori during pregnancy. 
Finally, the treatment of $\mathrm{H}$. Pylori infection may reduce the risk of hyperemesis gravidarum and its complications.

\section{REFERENCES}

1. Nasr AA, Aboulfoutouh I, Nada A, Younan MA, Saed M , El-Khayat W (2012): Is there an association between Helicobacter pylori infection and hyperemesis gravidarum among Egyptian women? Evidence Based Women's Health Journal, 2:100-103

2. Bailit JL (2005): Hyperemesis gravidarum: Epidemiologic findings from a large cohort. Am J Obstet Gynecol.;193(3 Pt 1):811-4.

3. Mansour GM ,Nashaat EH (2011): Role of Helicobacter pylori in the pathogenesis of hyperemesis gravidarum. Arch Gynecol Obstet., 284:843-847

4. Nanbakhsh F, Mohaddesi H, Bahadory F, Amirfakhrian J , Mazloomi P (2013): Comparison of Helicobacter pylori infection between pregnant women with hyperemesis gravidarum and controls. World Applied Sciences Journal, 28 (12): 1918-1922

5. Guven MA, Ertas IE, Coskun A, Ciragil P (2011): Serologic and stool antigen assay of Helicobacter pylori infection in hyperemesis gravidarum: Which test is useful during early pregnancy? Taiwanese Journal of Obstetrics \& Gynecology, $50: 37 \mathrm{e} 41$

6. Boltin D, Perets TT, Elheiga SA, Sharony A, Niv Y, Shamaly H, Dickman R (2014): Helicobacter pylori infection amongst Arab women with hyperemesis gravidarum - a prospective, controlled study. International Journal of Infectious Diseases, $29: 292-295$

7. Stenström B, Mendis A, Marshall B (2008): Helicobacter pylori-The latest in diagnosis and treatment. Aust Fam Physician, 37 (8): 608-12.

8. Kazerooni T, Taallom M, Ghaderi AA (2002): Helicobacter pylori seropositivity in patients with hyperemesis gravidarum. International Journal of Gynecology and Obstetrics, 79: 217-220

9. Jamal A, Pooransari P , Ansari R (2004): Relationship between Helicobacter pylori seropositivity and hyperemesis gravidarum, Acta Medica Iranica, 42: 367-370.

10. Jeffrey D, Quinlan JD , Hill A (2003): nausea and vomiting of pregnancy. Am fam. Physician, 68:121-8

11. Xia LB, Yang J, Li AB et al. (2004): Relationship between hyperemesis gravidarum and Helicobacter pylori seropositivity. Chin Med J., 117 (2):301-302

12. Wu CY, Tseng JJ, Chou MM, Lin SK, Poon SK, Chen GH (2000): Correlation between Helicobacter pylori infection and gastrointestinal symptoms in pregnancy. Adv Ther.,17:152- 8 .
13. Hayakawa S, Nakajima N, Karasaki-Suzuki M et al. (2000): Frequent presence of Helicobacter pylori genome in the saliva of patients with hyperemesis gravidarum. Am J Perinatol., 17:243-247.

14. Karaca C, Guler N, Yazar A et al. (2004): Is lower socioeconomic status a risk factor for Helicobacter pylori infection in pregnant women with hyperemesis gravidarum? Turk J Gastroenterol., 15: 86-89.

15. Loh K Y , Sivalingam N (2005): Understanding hyperemesis gravidarum. Med J Malaysia, 60 (3):394-9.

16. Jarraya A, Elleuch S, Zouari J, Trigui K, Sofiene A, Smaoui M, Kolsi K (2015): Hyperemesis gravidarum with severe electrolyte disorders: report of a case. The Pan $\boldsymbol{A}$ frican medical journal, 20: 264-264.

17. Li L, Li L, Zhou X, Xiao S, Gu H ,Zhang G (2015): Helicobacter pylori Infection Is associated with an increased risk of hyperemesis gravidarum: A Meta-Analysis. https://www.ncbi.nlm.nih.gov/pubmed/25861257

18. Goodwin TM (2008): Hyperemesis gravidarum. Obstet Gynecol Clin N Am., 35:401-417.

19. Sheehan $P$ (2007): Hyperemesis gravidarum--assessment and management. Aust Fam Physician., 36:698-701.

20. Fejzo MS, Macgibbon KW and Romero R (2011): Recurrence risk of hyperemesis gravidarum. J Midwifery Womens Health,56:132.

21. Frigo P, Lang C, Reisenberger KH , Hirschl AM (1998): Hyperemesis gravidarum associated with Helicobacter pylori seropositivity. Obstet Gynecol.,91:615-617.

22. Jacoby E , Porter K (1999): Helicobacter pylori infection and persistent hyperemesis gravidarum. Am J Perinatol., 16: 85-8.

23. Shirin H, Sadan O, Shevah O et al .(2004): Positive serology for Helicobacter pylori and vomiting in the pregnancy. Arch. Gynecol. Obestet., 270:10-14.

24. Delport W, van der A,Merwe SW (2007): The transmission of Helicobacter pylori: the effects of analysis method and study population on inference. Best Pract Res Clin Gastroenterol., 21 (2): 215-36.

25. Karadeniz R, Ozdegirmenci O , Altay M (2006): Helicobacter pylori seropositivity and Stool antigen in patients with hyperemesis gravidarum. Infect Dis Obstet Gynecol., 2006(1):73073.

26. Hoang TT, Wheeldon TU,Bengtsson C (2004): Enzymelinked immunosorbent assay for Helicobacter pylori needs adjustment for the population investigated. J Clin Microbiol., 42:627-30

27. Mai E, Abo Seada M, Shafik A , Mamdouh A (2014): Role of the Helicobacter pylori in the pathogenesis of hyperemesis gravidarum. MsC thesis, Ain Shams Faculty of Medicine, Department of Obstetrics and Gynecology. 